International Journal of Biology, Pharmacy and Allied Sciences (IJBPAS) 'A Bridge Betueen Caboratory and QRendo' WwW.iibpas.com

SYNTHESIS AND APPLICATIONS OF SILVER NANOPARTICLES: A REVIEW

\title{
GOYAL $\mathrm{S}^{1}$, ANDHARE $\mathrm{P}^{2}$, MARCHAWALA $\mathrm{F}^{2}$, BHATTACHARYA ${ }^{2}$ AND UPADHYAY $\mathbf{D}^{2 *}$
}

1: Student, M. Sc. Microbiology, Parul Institute of Applied Sciences, Parul University, Post Limda, Waghodia, Gujarat, 391760

2: Assistant Professor, Parul Institute of Applied Sciences, Parul University, Post Limda, Waghodia, Gujarat, 391760

*Corresponding Author: Dr. Dhwani Upadhyay; E Mail:dhwani.updhyay82123@paruluniversity.ac.in; Tel: +919558021474

Received 22 ${ }^{\text {nd }}$ Jan. 2021; Revised 24 ${ }^{\text {th }}$ Feb. 2021; Accepted 25 ${ }^{\text {th }}$ March 2021; Available online $1^{\text {st }}$ April 2021 https://doi.org/10.31032/IJBPAS/2021/10.4.1041

\section{ABSTRACT}

In more recent times Nanotechnology has become a hotbed of research. Nanotechnology are associated with nanoparticles with a thickness of 1-100 $\mathrm{nm}$ on one side, that is used in medical chemicals, quantum optics, and other fields. Nanoparticles are often used due to its light weight, shape, or structure, that are shown to modify the performance of any other entity which makes contact with it. Organic, physiological, and ecological approaches can all be used to repair these particles. Chemical, physical, and environmental approaches can all be used to repair these particles. Researchers have been interested in silver nanoparticles (NPs) because of their unusual properties (e.g., size and shape depending on optical, antimicrobial, and electrical properties). Various methods for preparing silver NPs for consolidation have been recorded in this review; notable examples include Laser light, nuclear irradiation, radiation generated by electromagnetic waves irradiation, electrochemical treatment, photochemical approaches, microwave processing, and biological methods are some of the techniques that have been used.

Keywords: Nanoparticle synthesis, Silver nanoparticles, physical synthesis 


\section{INTRODUCTION}

Nanotechnology is an area of knowledge and transformation involving the analysis of materials in the nanoscale, usually between 1 and 100 nanometers. It is a nanoscale science that relates to various fields of science such as dentistry, pharmaceuticals, and bioengineering, material science, agriculture, food service, cosmetic, clinical, and diagnostic applications [1].

Nanobiotechnology, bio nanotechnology, and nanobiology are concepts used to describe the synthesis of nanotechnology and biology. Polyphenols, flavonoids, tannins, and phenolic compounds are among the bioactive compounds found in plants and plant metabolites [2].

With their distinct mode of action, AgNPs nanoparticles have the most impactful outcomes of all nanotechnological applications. In the last decade, the use of metals as antimicrobial agents has been shown to have good scientific support. In certain cases, scientists discovered that metal ions closely bound to antibiotic structures (e.g., bacitracin, erythromycin, and cephalexin) handle biocidal action; they have been attached to the antibiotic molecule without altering the antibiotic structure, but they have increased the activity [3]. In comparison to other metals, silver nanoparticles have a stronger antibacterial impact. Green nanoparticle synthesis has been achieved using environmentally acceptable plant extract and eco- friendly reducing and capping agent [4]. A transmission electron microscope (TEM), X-ray diffraction (XRD), and Fourier transfer infrared spectroscopy were used to examine the morphology and structure of AgNPs (FTIR). In turn, the antibacterial activity of silver nanoparticles was assessed to use a broth dilution test against $S$. aureus and $E$. coli. To improve the bactericidal activity of silver nanoparticles, a calculation was performed with various types of silver NPs generated at various annealing temperatures and $\mathrm{pH}$ levels. [5].

\section{NANOPARTICLES}

A nanoparticle is a very small unit with a diameter of one nano meter. Nanoparticles can be found in nature and are often generated as a result of human activity. They have distinct properties due to their submicroscopic scale, and the nanoparticles generated can be used in active applications in a variety of fields Pharmaceuticals, engineering catalysis, and environmental change are all examples of this [6]. Nanoparticles can be made from a wide range of industrial chemicals, namely metals, carbonates, semi ceramic ingredients, polyethylene, environmental, carbon, and organic compounds. Nanoparticles come in a range of shades, 
like spheres, cylinders, platelets, and tubes

[7]. Nanoparticles are usually designed to fulfil the particular needs of end applications [8].

\section{NANO SILVER}

Silver is one of the materials used in nano formulation (nano silver). Silver is used in filtration filters for drinking water and swimming pools because of its antimicrobial properties. Silver and metal have been ultrafine particles in many ways to generate nano silver, including sparks, reducing electrochemical, irradiation solution, and cryo-chemical synthesis [9]. Nano silver particles have a diameter of less than 100 nanometers and contain between 20 and 15,000 silver atoms. Nanostructures may also be made into tubes, strings, multifaceted structures, or movies. The silver particles have deformed physic-chemical deviations on a nanoscale scale (such as $\mathrm{pH}$ depending on the separation of solid and dissolved matter) [10]. This is because each mass has a wide surface area, allowing a large number of atoms to interact with the atmosphere. Nano silver is now being used in rising consumer value and medical devices due to silver structures at the nanoscale. Since silver has a soft white sheen, silver nanoparticles are commonly used to give items a silver sheen [11].

\section{PRODUCTION OF SILVER NANOPARTICLE}

\section{Physical approaches}

The most effective methods of the body are dehydration and laser removal. Physiological processes have advantages over chemical processes in terms of the absence of solvent contamination in small prepared films and the similarity in NP distribution [12]. The use of a furnace to combine silver NPs with air pressure has some disadvantages, such as taking up a lot of space, using a lot of energy while increasing the natural temperature around the source wells, and taking longer to achieve thermal stability. Furthermore, a traditional furnace takes more than a few kilowatts of power and a few minutes of heating time to achieve a stable temperature [13]. It has been demonstrated that silver nanoparticles can be used in conjunction with a small clay heater to create a local temperature. The springs were drained using a small clay heater. Since the gradient in a hot object is much colder than in a boiler, steam fumes will cool to a degree [14].

This allows for the production of small NPs with a high density. Since particle temperature does not vary with time, particle generation is very stable. This method of observation may be used as a nanoparticle generator for long-term experiments in inhalation toxicity studies, as well as a nanoparticle measuring tool [15]. According to the findings, global 
warming increased the geometric scale, geometric deviations, and the total number of filters for NPs. Even at high temperatures, round NPs can be found without undergoing integration [16].

Elimination of metal tools may be paired with silver NPs. Many factors influence the ablation efficiency and characteristics of the nano-silver particles generated, including laser duration, including the metal target, length of laser pulses (femto-, pico-, and nanosecond), laser fluence, and the distance between the active liquid and the extraction rate, with or without surfactants. [17].

\section{Chemical approaches}

Chemical reduction by natural and biological agents is a popular method of integrating silver NPs. To reduce silver ions $(\mathrm{Ag}+)$ in solid or non-aqueous solutions, various reducing agents such as sodium citrate, ascorbate, sodium borohydride (NaBH4), elemental hydrogen, polyol phase, Tollens reagent, N, Ndimethylformamide (DMF), and poly (ethylene glycol) -block copolymers are used [18]. These reducing agents reduce Ag + and cause silver and iron $(\mathrm{Ag} 0)$ to form, which are then synthesised into oligomeric clusters. Silver and metal particles are associated with the formation of these collections. To avoid interaction with the nanoparticle surfaces, it is important to use protective materials to stabilise the released
NPs mostly during preparation of nanoparticle metal, as well as to avoid NPs from entering or binding to the particles surface [19]. Active surfactants (such as sodium salts, amines, chemicals, and hard liquor) interacting with particle surfaces can help to stabilise particle growth and prevent particles from catching, crashing, or weakening top layer. The most popular polymeric chemicals have been reported to be poly (vinyl alcohol), poly (vinylpyrrolidone), poly (ethyleneglycol), poly (methacrylic cacid), and polymethylmethacrylate. Agents that are extremely efficient at strengthening [20]. At ambient temperature, silver NPs can be made by combining the corresponding iron ions with reduced polyoxometalates, which serve as reducing and stabilising agents. Polyoxometalates are water soluble and have powerful multielectron redox reactions that do not interfere with their formation [21].

\section{Biological approaches}

Researchers have spent years developing environmentally sustainable materials to minimise, position, and reinforce agents used to prepare silver nanoparticles as morphology and scale. [22]. Without the use of volatile, toxic, or costly chemical agents, biological methods can be used to assemble silver nanoparticles. The reduction of iron by biomolecule compounds found in the extraction of 
certain substances (e.g., enzymatic, glutamic acid, disaccharides, and vitamins) is not environmentally hazardous, but it is chemically complex [23].

\section{APPLICATION OF SILVER NANOPARTICLES}

Nanomedicine is a vast field of study with enormous potential for improving human diagnostic accuracy and care. Dispersed nanoparticles are commonly used in nano biomedicine as illuminated biological labels, medications, and biochemical delivery agents, as well as in facilities like pathogen bio detection, synthetic biology, plant destruction with fever (hyperthermia), enhanced MRI comparison, and phagokinetic studies [24]. Researchers have begun studying biosynthesized nanoparticles in programmes such as targeted delivery, treatment for cancer, molecular therapy and DNA testing, antimicrobials, biosensors, improved reaction rates, isolation science, and MRI, despite the fact that the area of biosynthesized nanoparticles is still relatively new. We've included several examples to demonstrate how these applications can be used [25].

Table 1: Application of silver nanoparticles

$\left.\begin{array}{|c|c|c|c|}\hline \text { Drug delivery } & \begin{array}{c}\text { M Magnetic nanoparticles are used to treat cancer. } \\ \text { Nanocarriers have enhanced pharmacodynamics and oral } \\ \text { bioavailability of therapeutics due to their high surface area to } \\ \text { volume ratio. }\end{array} & \begin{array}{c}\text { They make hydrophobic compounds more soluble and thus ideal for } \\ \text { transdermal delivery. }\end{array}\end{array}\right]$

\section{CONCLUSION}

Because of their appealing appearance, nanomaterials play a significant role in pathology and regenerative medicine. A new survey showed that using a renewable, relatively low reducing agent or a well green nano science technique, metal nanocrystals can be produced without the use of harmful solvents or waste.

\section{ACKNOWLEDGEMENT}

It's our privilege and honour to express our sincerest gratitude to the Parul University, 
Vadodara, Gujarat for providing me all the necessary support and facilities including state of the art infrastructural facilities with advanced technological scientific laboratories and everything else that was required to carry out this work.

\section{REFERENCE}

[1] People. Nature Biotechnology. 10 October 2007: 1186-1186.

[2] Anjum, D. H. Characterization of Nanomaterials with Transmission Electron Microscopy. IOP Conference Series: Materials Science and Engineering. August 2016: 012001.

[3] Hussain, Imtiaz et al. Green Synthesis of Nanoparticles and Its Potential Application. Biotechnology Letters 4, December (2015): $545-560$.

[4] Horikoshi, Satoshi, and Nick Serpone. Introduction to Nanoparticles. Microwaves in Nanoparticle Synthesis. WileyVCH Verlag GmbH \& Co. April 2013 1-24.

[5] Berry, Catherine C. Applications of Inorganic Nanoparticles for Biotechnology. Nanobiotechnology - Inorganic Nanoparticles vs Organic Nanoparticles. Elsevier, July2012. 159-180.

[6] Iravani, Siavash. Bacteria in Nanoparticle Synthesis: Current
Status and Future Prospects. International Scholarly Research Notices, October 2014: 1-18.

[7] Kuppusamy, Palaniselvam et al. Biosynthesis of Metallic Nanoparticles Using Plant Derivatives and Their New Avenues in Pharmacological Applications An Updated Report. Saudi Pharmaceutical Journal 4 September 2016: 473-484.

[8] Lara, Humberto $\mathrm{H}$ et al. Silver Nanoparticles Are Broad-Spectrum Bactericidal and Virucidal Compounds. Journal of Nanobiotechnology 1. March 2011: 30.

[9] Gong, Ping et al. Preparation and Antibacterial Activity of $\mathrm{Fe}_{3} \mathrm{O}_{4} @ \mathrm{Ag}$ Nanoparticles. Nanotechnology. June 2007: 285-604.

[10] Mohanpuria, Prashant, Nisha K. Rana, and Sudesh Kumar Yadav. Biosynthesis of Nanoparticles: Technological Concepts and Future Applications. Journal of Nanoparticle Research 3 October 2007: 507-517.

[11] Dykman, Lev, and Nikolai Khlebtsov. Immunological Properties of Gold Nanoparticles. Gold Nanoparticles in Biomedical Applications. CRC Press, December 2017. 241-278. 
[12] Zinjarde, Smita Sachin. BioInspired Nanomaterials and Their Applications as Antimicrobial Agents. Chronicles of Young Scientists 1. August (2012): 74.

[13] Oliveira, Marcela M. et al. Influence of Synthetic Parameters on the Size, Structure, and Stability of DodecanethiolStabilized Silver Nanoparticles. Journal of Colloid and Interface Science 2, December (2005): 429 435.

[14] Siegel, Jakub et al. Progressive Approach for Metal Nanoparticle Synthesis. Materials Letters, December (2012): 47-50.

[15] Vauthier, Christine. Methods for the Preparation of Nanoparticles by Polymerization. Polymer Nanoparticles for Nanomedicines. Springer International Publishing, 2016. 123-157.

[16] Feng, Pengfa, and Weicheng Cao. Properties, Application and Synthesis Methods of NanoMolybdenum Powder. Journal of Materials Science and Chemical Engineering 09, 2016: 36-44.

[17] Nanoparticle Synthesis. Nanoparticulate Materials. John Wiley \& Sons, Inc., October 2012. 24-127.
[18] Thakkar, Kaushik N., Snehit S. Mhatre, and Rasesh Y. Parikh. Biological Synthesis of Metallic Nanoparticles. Nanomedicine: Nanotechnology, Biology and Medicine 2 April (2010): 257262.

[19] Klein, Sylke, Markus Winterer, and Horst Hahn. Reduced-Pressure Chemical Vapor Synthesis of Nanocrystalline Silicon Carbide Powders. Chemical Vapor Deposition 04. July (1998): 143149.

[20] Herren, Markus et al. Luminescence and Surface Effects of Closed-Shell Transition Metal Ions in Sol-Gel Silica Glasses. Journal of Luminesce. June (1997): 417-418.

[21] Yu, Zhong Qing et al. Blue Photoluminescent Properties of Pure Nanostructured $\gamma-\mathrm{A} 12 \mathrm{O} 3$. Journal of Materials Research. July (2001): 1890-1893.

[22] Gupta, Amit, and Simon Silver. Molecular Genetics: Silver as a Biocide: Will Resistance Become a Problem? Nature Biotechnology. October (1998): 888-888.

[23] Santhosh Kumar, Thirunavukkarasu et al. Synthesis of Silver Nanoparticles Using Nelumbo Nucifera Leaf Extract 
and Its Larvicidal Activity against

Malaria and Filariasis Vectors.

Parasitology Research 3, October (2010): 693-702.

[24] Panyala, Nagender Reddy, Eladia María Peña-Méndez, and Josef Havel. Silver or Silver Nanoparticles: A Hazardous Threat to the Environment and Human Health? Journal of Applied Biomedicine 3, July (2008): 117 129.

[25] Saifuddin, N., C. W. Wong, and A. A. Nur Yasumira. Rapid Biosynthesis of Silver Nanoparticles Using Culture Supernatant of Bacteria with Microwave Irradiation. E-Journal of Chemistry 1, (2009): 61-70.

[26] Chaloupka, Karla, Yogeshkumar Malam, and Alexander $\mathrm{M}$. Seifalian. Nanosilver as a New Generation of Nanoproduct in Biomedical Applications. Trends in Biotechnology. November (2010): 580-588

[27] Fan, Tong-Xiang, Suk-Kwun Chow, and Di Zhang. Biomorphic Mineralization: From Biology to Materials. Progress in Materials Science 5, September (2009): 542-659.

[28] Fortini, Otavio Macedo. Energy and Raw Materials in the Selection of Technologies for Iron and Steel. Metallurgical and Materials Transactions E 3, May (2016): 189-202.

[29] Wong, Kenneth K.Y. et al. Topical Delivery of Silver Nanoparticles Reduces Systemic Inflammation of Burn and Promotes Wound Healing. Nanomedicine: Nanotechnology, Biology and Medicine 4 December (2006): 306. 